\title{
Deux entrepreneurs schumpétériens : la Compagnie Ansaldo navale-mécanique des frères Bombrini et les marchés étrangers entre le $19^{\mathrm{e}}$ et le $20^{\mathrm{e}}$ siècles
}

\author{
Two Schumpeterian entrepreneurs: The Bombrini brothers' Ansaldo \\ company and foreign markets between the 19th and 20th centuries
}

\author{
Paola Nardone ${ }^{1}$, Natascia Ridolfi ${ }^{2}$ \\ ${ }^{1}$ Université de Chieti, Italie \\ ${ }^{2}$ Université de Chieti, Italie
}

RÉSUMÉ. L'article analyse la politique d'entreprise de la société Ansaldo de Gênes sous la direction des frères Bombrini, entrepreneurs innovants et dynamiques. Leur direction a placé Ansaldo au sommet de la production navale et mécanique nationale. Ce secteur, particulièrement stratégique à la fin du XIXe et au début du XXe siècles, nécessitait des investissements massifs, qui étaient prévus pour réaliser des projets visant non seulement à réaliser une structure verticale du processus de production, mais aussi à permettre à Ansaldo de mesurer sa propre capacité dans un secteur spécialisé tel que l'industrie navale-mécanique. Les frères Bombrini ont commencé à se tourner vers les marchés étrangers afin de créer des nouvelles perspectives de succès pour les produits de l'entreprise. Cette décision a été prise surtout compte tenu des effets de plus en plus négatifs que la crise avait dans le secteur de la métallurgie, provoquée par le désengagement progressif du gouvernement dans les commandes militaires. Cet article repose sur l'étude de la documentation des archives et d'une bibliographie dense.

ABSTRACT. This article analyzes the company policy of Ansaldo, a Genoese business under the direction of the Bombrini brothers, both innovative and dynamic entrepreneurs. Their leadership placed Ansaldo at the top of national naval and mechanical production. This sector, which was particularly strategic in the late 19th and early 20th centuries, required massive investment to carry out projects aimed not only at achieving a vertical structure in the production process, but also at allowing Ansaldo to measure its own capacity in a highly specialized sector such as the naval-mechanical industry. The Bombrini brothers began to turn their attention to the foreign market in order to create new opportunities for product success. This decision was made primarily because of the increasingly negative effects of the crisis in the metalworking sector, caused by the progressive disengagement of the government in military orders. This article was prepared using archive documentation and an extensive bibliography.

MOTS-CLÉS. Industrie, Entrepreneur, Innovation, Italie, Expansion commerciale.

KEYWORDS. industry, entrepreneur, innovation, Italy, commercial expansion.

\section{Introduction}

L'Italie est entrée dans l'industrialisation plus tard que les pays nord-européens, mais elle n'en compte pas moins des entrepreneurs dynamiques qui à partir de la fin du XIXe siècle ont joué un rôle important au niveau de l'économie du pays, comme à l'échelle internationale. La société Ansaldo des frères Bombrini est l'une d'entre elles. Mais, si l'industrialisation de l'économie italienne a été portée par des capitaines d'industrie, l'Etat a fortement soutenu ce processus, généralement en lien avec les premiers. La réforme douanière de 1878 marqua en effet le lancement d'une politique protectionniste fortement influencée par la pression du front industriel des régions du nord de l'Italie, représenté alors surtout par les groupes textiles de la Vénétie. En réalité, il s'agissait d'une réforme technique plutôt que d'un réel changement d'orientation de la politique commerciale du gouvernement italien. En fait, la transition vers des droits ad valorem assurait une couverture modérément plus protectionniste en faveur des industries déjà consolidées, telles que les textiles, en 
particulier la filature et le tissage du coton, de la laine et de la soie soutenues par des industriels puissants ${ }^{1}$.

Cette réforme, que naissait en raison des nombreux compromis entre les différentes forces politiques présentes au Parlement, introduisait des tarifs modestes sur les importations de blé et de céréales, sans aucune protection pour les industries émergentes, comme la mécanique ${ }^{2}$. Malgré cela, en un peu plus d'un an, les tarifs protectionnistes, fondés principalement sur des tarifs spécifiques et appliqués dans toute Europe, ne représentaient pas un obstacle au commerce international dans un contexte général de baisse des prix. Dès lors, l'année 1879 pouvait être considérée, sur la base des séries historiques les plus récentes, comme «l'année seuil du processus d'industrialisation ». Ainsi commença un cycle de croissance industrielle (1879-1887) avec un taux annuel moyen d'environ $8 \%$, bien qu'avec « des fortes fluctuations et différenciations sectorielles $»^{3}$.

L'intervention par l'État pour stimuler la croissance économique du pays a également été mise en œuvre à travers une série de mesures législatives en faveur de la grande industrie mécanique (équipements militaires et construction ferroviaire), secteur qui a alors le plus largement bénéficié de la nouvelle politique économique du gouvernement. Vers le milieu des années 1880 des stratégies industrielles de substitution aux importations ont été développées, en particulier dans le secteur de la mécanique ferroviaire ${ }^{4}$, grâce à l'initiative d'entreprises fortement spécialisée (comme

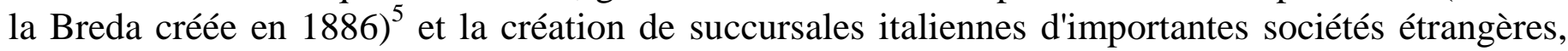
dont le meilleur exemple est la société de construction mécanique de Saronno, une filiale de la société allemande Maschinenfabrik Esslingen (installée à Stuttgart), intéressée par le marché ferroviaire italien après la ratification, en 1885, des conventions conclues l'année précédente avec trois grandes compagnies ferroviaires ${ }^{6}$, par le gouvernement. Cette disposition donnait des avantages fiscaux aux entreprises qui avaient commandé des fournitures à des usines nationales. La situation favorable de l'industrie ferroviaire a également stimulé la compagnie Ansaldo qui a contribué à relancer la production de matériel roulant, qui avait été interrompue en 1871. Durant les vingt années entre les mesures législatives de 1885 et la nationalisation du réseau ferroviaire en 1905, Ansaldo a construit 290 locomotives, ce qui place l'entreprise à la deuxième place au niveau national, immédiatement après Breda, mais à une distance considérable de Saronno ${ }^{7}$.

Malgré les bons résultats obtenus dans la construction ferroviaire, la situation favorable de l'économie italienne dans les années 1880 avait avant tout profité à la construction navale, en particulier à Ansaldo dont la contribution a été un facteur décisif pour le démarrage de l'industrie mécanique au cours des vingt dernières années du XIXe siècle. La loi n. 3587 du 6 décembre 1885 visait le développement des interventions publiques en faveur de la marine marchande et des chantiers navals ${ }^{8}$. A cette date, les usines les plus importantes se trouvaient à Livourne (Cantiere Orlando), dans la région napolitaine (Cantieri Navali Thomas R. Guppy, John Pattison et Armstrong Mitchell et C.) et dans l'arrière-pays génois, en particulier à Sestri Ponente avec les chantiers navals de la société Odero e Ansaldo.

\footnotetext{
${ }^{1}$ Le groupe était dirigé par Alessandro Rossi « le grand lainier de Schio pour qui le libéralisme n'était rien d'autre que le protectionnisme des forts », F. Amatori, A. Colli, Impresa e industria in Italia dall'Unità a oggi, Venise, Marsilio, 1999, p. 44.

${ }^{2}$ P. Ciocca, G. Toniolo, Storia economica d'Italia, 2, Annali, Bari, Laterza, 1999, p. 131-132.

3 Ivi, p. 133.

${ }^{4}$ M. Lungonelli, L'Ansaldo e l'industria, cit., p. 57-58; M. Merger, Un modello di sostituzione: la locomotiva italiana dal 1850 al 1914, in «Rivista di Storia Economica», (III), n. 1, p. 66-105, 1986 e id. L'industrie italienne de locomotives, reflet d'une industrialisation tardive et difficile (1850-1914), in «Histoire Economie et Société», n. 3, p. 335-370, 1989.

${ }^{5}$ M. Lungonelli, L'Ansaldo e l'industria, cit., p. 57.

${ }^{6}$ L'étude la plus stimulante et la plus complète sur l'activité de l'entreprise Maschinenfabrik Esslingen in Italie est l'essai par P. Hertner, Capitale tedesco e industria meccanica in Italia: la Esslingen a Saronno, in «Società e Storia», n. 17, p. 583-621, 1982.

${ }^{7}$ M. Merger, Un modello di sostituzione, cit., pp. 87-88 e tab. A2, M. Lungonelli, L'Ansaldo e l'industria, cit., p. 59.

${ }^{8}$ G. Barone, Lo Stato e la Marina Mercantile in Italia (1881-1882) in «Studi Storici», n. 3, p. 624-659, 1974.
} 
Cette mesure a été conçue avec le but de soutenir les activités de construction navale et de stimuler, avec des avantages fiscaux appropriés, les investissements des entreprises italiennes et étrangères. Elle prévoyait des primes de navigation pour les navires construits à l'étranger, afin de ne pas pénaliser «les armateurs qui trouvaient plus commode de se tourner vers les chantiers navals anglais pour moderniser leurs flottes et étendaient les primes de production aux voiliers en bois, qui au départ ils n'auraient pas dû recevoir de subventions, en raison des vives protestations des propriétaires de petits chantiers $\gg$.

Dans le cadre de cet article, notre objectif est de présenter les résultats d'une étude historique que nous avons réalisée à partir d'archives de la société Ansaldo des frères Bombrini, une entreprise dirigée par les frères Bombrini eux-mêmes, des entrepreneurs schumpétériens, dans un secteur industriel nouveau, celui du transport ferroviaire et naval, puis dans l'industrie de l'armement. L'entreprise a été créée à Gênes en 1853, avant l'unification italienne et s'est, pour la plupart, développée à l'international, grâce à des innovations techniques et organisationnelles, mais également à des collaborateurs compétents et zélés. Nous expliquerons dans un premier temps comment la société s'est positionnée dans des industries nouvelles en Italie, puis à l'international, d'abord en Amérique latine et au Mexique, puis en Europe du Nord, notamment en Belgique et en Angleterre, pour contrecarrer le ralentissement de l'activité industrielle sur le marché italien.

\section{Une stratégie d'entreprise tournée vers des constructions nouvelles (1883-1892)}

Dans le cycle de croissance industrielle (1879-1887) qui vient d'être esquissé, la première phase d'expansion d'Ansaldo dans les années 1883-1892 permit à l'entreprise génoise de s'insérer dans le tissu industriel italien. En mars 1882, Carlo Bombrini meurt. Il était le dernier associé vivant de la société en commandite, fondée en 1853 par les Génois Giovanni Ansaldo (qui a donné son nom à l'entreprise), professeur de géométrie à l'Université de Gênes et de mécanique appliquée à l'école technique du soir de la Chambre de commerce de Gênes, l'entrepreneur Giacomo Filippo Penco, l'armateur Raffaele Rubattino et Carlo Bombrini, directeur de la Banque Nationale ${ }^{10}$. Nous ne nous attarderons pas ici sur les trente premières années de la vie d'Ansaldo qui ont déjà nourri une historiographie très complète ${ }^{11}$. Quand les frères Giovanni (1835-1924) et Carlo Marcello Bombrini (1835-1909) héritent de l'entreprise en 1883 celle-ci paraît particulièrement difficile à diriger, mais ils obtiennent néanmoins de bons résultats entre 1883 et 1892. Déjà dans le rapport publié en 1884 par la société pour la période de deux ans 1883-1884, les frères Bombrini soulignaient « fièrement que la valeur annuelle des productions avait plus que doublé » par rapport aux trente années précédentes, grâce à de nouveaux équipements ${ }^{12}$. Lors de l'Exposition générale italienne de Turin en 1884, ils avaient fait connaître le potentiel de croissance de l'entreprise dans de nombreux secteurs, notamment le génie naval et les chemins de fer. En fait, l'installation d'un nouvel atelier était déjà en cours pour l'assemblage et la mise en service de 36 locomotives par an, soit environ la moitié des besoins nationaux annuels. Par ailleurs, la loi d'avril 1885, avec la réforme des concessions ferroviaires, poussa les frères Bombrini à faire de nouveaux investissements massifs dans la construction d'une usine capable de fabriquer environ 200 locomotives par an. L'objectif fut atteint en 1887.

Dans le même temps, la croissance importante de la capacité de production de la construction ferroviaire, ainsi que la fluctuation des commandes, avaient empêché d'« atteindre un équilibre rentable entre les coûts fixes et variables et avait considérablement réduit la compétitivité des

\footnotetext{
${ }^{9}$ M. Doria, L'Ansaldo. L'impresa e lo Stato, Milan, F. Angeli, 1989, p. 41.

${ }^{10}$ F. Amatori, A. Colli, Imprese e industria, cit., p. 33-34.

11 À cet égard, veuillez voyer les sages in G. Mori (édité par), Storia dell'Ansaldo. 1. Le origini 1853-1882, Bari, Laterza, 1994.

12 Le titre complet de la publication est Notizie sullo stabilimento meccanico e di costruzioni navali Gio Ansaldo e C. di Sampierdarena con breve cenno sugli oggetti da esso presentati alla Esposizione Generale di Torino, Turin, 1884.
} 
entreprises opérant dans le pays, en ralentissant le processus de substitution aux importations» ${ }^{13}$. L'industrie de la construction navale constituait pour les frères Bombrini un choix stratégique vital. On devait tester le potentiel de l'entreprise et justifier les investissements massifs dans des projets visant non seulement à créer une structure de production intégrée, mais aussi à permettre à Ansaldo d'évaluer ses compétences dans un secteur très spécialisé encore presque inexploré par la jeune classe entrepreneuriale italienne. Il était urgent de restructurer en profondeur la marine marchande. Dans cette perspective, la loi n. 3547 du 6 décembre 1885 parut aux armateurs italiens comme le signe avant-coureur d'un développement à venir d'une politique protectionniste de la construction navale ${ }^{14}$.

Dans ce contexte, la compagnie Ansaldo des frères Bombrini a pu, dans les années 1886-1892, occuper une position de leader, en utilisant également le chantier naval modernisé de Sestri Levante, loué en 1866 par les frères Cadenaccio. Cependant, les commandes de navires des particuliers sont restées modestes et n'auraient pas justifié les investissements réalisés au cours de la décennie par la compagnie Ansaldo si entre-temps elles n'avaient pas été accompagnées de commandes d'Etat beaucoup plus rentables. La société a été poussée à accepter ces commandes. Elle y fut également incitée par l'installation de Benedetto Brin au département de la Marine (le 30 mars 1884) ${ }^{15}$. Le nouveau ministre était le porte-étendard de l'orientation protectionniste et de la politique en faveur d'une grande flotte nationale, de guerre et marchande, ainsi que des industries lourdes, en particulier la sidérurgie. Il estimait que l'initiative privée était indispensable et il a fait valoir la nécessité "de disposer de unités blindées puissantes, d'un gros tonnage comme condition essentielle pour que le pays faisait une politique de puissance ». Dans cette optique et avec ces objectifs, la relation de Brin avec Vincenzo Stefano Breda a mûri et s'est développée. Ce dernier était alors à la tête d'une grande entreprise, la Veneta, dirigée avec un groupe de banquiers et d'hommes d'affaires de sa région. Ils étaient notamment engagés dans les marchés publics. Breda avait déjà élaboré une série de projets pour doter le pays d'un système adéquat d'usines de guerre. L'industrie Terni était pratiquement contrôlée par les Vénitiens, l'usine avait été construite dans la ville ombrienne car elle était éloignée de la mer et des frontières, donc très difficile à attaquer en cas de guerre ${ }^{16}$.

En réalité, les commandes confiées à Ansaldo demeurèrent modestes, car la construction des navires de grand tonnage était essentiellement le fait des arsenaux militaires. Néanmoins, les commandes ont été assez rentables et ont développé une nouvelle version de petites et très petites unités navales, à savoir les croiseurs, les destroyers et les remorqueurs. Cela a permis au nouveau chantier naval de Sestri Ponente de se tailler une part substantielle des commandes militaires ${ }^{17}$. Malgré cette intéressante participation de la Compagnie Ansaldo au renforcement de la flotte militaire, les plus importantes compagnies maritimes de l'époque, Odero et Orlando, sont restés les partenaires privilégiés pour l'État. Cependant, les frères Bombrini rapportaient la construction de 18 des 93 torpilleurs du type Schichau pour de petits navires militaires entre 1886 et 1891. La marine royale avait, entre-temps, acheté la société homonyme d'Elbing (une ville de Prusse orientale, à

\footnotetext{
13 A. M. Falchero, Le strutture e l'evoluzione dell'Ansaldo, in Storia dell'Ansaldo. 2, cit.., p. 30-31.

14 Ivi, p. 31.

15 Ivi, p. 32.

${ }^{16}$ F. Amatori. A. Colli, Impresa e industria in Italia, cit., p. 44-46. Pour une histoire exhaustive de la Compagnie Terni, F. Bonelli, Lo sviluppo di una grande impresa in Italia. La Terni dal 1884 al 1962, Turin, Einaudi, 1975, à intégrer avec l'essai par S. Licini, Dall'Elvetica alla Breda. Alle origini di una grande impresa milanese (1846-1918), in «Società e Storia», n. 63, pp. 79123, 1994, et AA. VV., La Breda. Dalla Società Italiana Ernesto Breda alla Finanziaria Ernesto Breda 1886-1986, Milan, Amilcare Pizzi Editore, 1986.

17 A. M. Falchero, Le strutture e l'evoluzione dell'Ansaldo, cit., p. 33. Les réflexions par L. De Rosa sur les dépenses de guerre pour la Marine restent d'actualité, Difesa militare e sviluppo economico in Italia (1861-1914), in L. De Rosa, La rivoluzione industriale in Italia e il Mezzogiorno, Rome-Bari, Laterza, 1973. Pour plus d'informations, voir les ouvrages publiés par le directeur du Génie Naval Antenore Bozzoni, Relazione della Commissione per le industrie meccaniche e navali, Rome, Tipografia Fratelli Bencini, 1885, Id., Relazione a Sua Eccellenza e il mare. Costruzioni navali, trasporti e commercio tra XV e XX secolo, Rome, Tipografia Fratelli Bencini, 1889, à intégrer avec G. Bozzoni, Marina militare e costruzioni navali, in Cinquant'anni di storia italiana, Milan, Hoepli, 1911, p. 1-73.
} 
l'époque allemande, annexée à la Pologne en 1945). Au cours de la même période, Ansaldo s'est vu confier la construction d'un des 7 croiseurs protégés de la classe Regioni, le Liguria et de 1 des 8 croiseurs de la classe Partenope, précisément le Minerva ${ }^{18}$.

\section{La stratégie d'expansion internationale des frères Bombrini (1893-1903)}

Entre 1883 et 1892, la Compagnie Ansaldo avait réussi à obtenir beaucoup de commandes de moteurs de marine de moyenne puissance, si bien que ceux-ci représentèrent alors la plupart (plus de 58\%) de sa chiffre d'affaires militaire. A l'inverse, la construction des croiseurs et de cuirassés, plus important d'un point de vue financier, stagnait en dépit de la commande du croiseur Garibaldi en mars 1893. Ce contrat fut rompu suite aux trois années noires de la crise économique internationale. Bien que peu nombreuses, les données de la Compagnie Ansaldo mettent en évidence l'impact violent de cette crise sur l'entreprise : 800 salariés sur 1600 ont été licenciés dans l'usine mécanique entre 1890 et 1893 et 520 sur 900 dans le chantier naval. Le blocage de la construction de locomotives dura jusqu'en 1895. L'entreprise enregistra des bénéfices de 47000 lires, alors que les désinvestissements s'élevaient à plus de 800000 lires $^{19}$. Cette situation d'ensemble, aussi négative qu'inquiétante, fut dénoncé sans relâche, dans de vibrantes protestations des dirigeants d'Ansaldo, envers les gouvernements italiens ${ }^{20}$.

En fait, la compagnie Ansaldo des frères Bombrini focalisait déjà son attention sur la nécessité de procéder à des changements, structurels et managériaux, profonds et rapides pour faire face aux processus de concentration en cours dans les industries maritimes italienne et étrangères. Le point de départ de la réflexion des Bombrini fut la contraction notable des dépenses militaires italiennes, notamment navales, après la crise ${ }^{21}$. Au cours de la décennie suivante, les relations de la Compagnie Ansaldo avec la marine royale furent, pour ainsi dire, sui generis, car elle avança à la société génoise les fonds nécessaires à la construction de navires à destination de l'étranger, en particulier les unités de la classe Garibaldi. Une telle procédure accentuait la rivalité et les rapports de concurrence féroces entre les principales compagnies maritimes du pays et, dans le même temps, exacerbait la controverse concernant les liens entre les entreprises privées et le ministère de la Marine. Cette situation, cependant, avait aussi ses aspects positifs, car la pratique adoptée avait offert aux armateurs italiens l'opportunité de profiter des tensions politiques et militaires entre les différents pays étrangers, atténuant ainsi au moins partiellement la réduction des dépenses militaires intérieures.

À ce stade, le choix prise par les frères Bombrini de se tourner vers le marché étranger était une chose inévitable, compte tenu des effets négatifs du désengagement de plus en plus important par le gouvernement. La recherche de nouveaux marchés mettait en évidence les caractéristiques entrepreneuriales pré-schumpétériennes que les frères Bombrini se sont appropriées ; éléments décisifs de leur réussite. Ils possédaient les qualités et les exigences du modèle de l'entrepreneur qui a été théorisé par Joseph Alois Schumpeter une décennie plus tard (1912) ${ }^{22}$. L'économiste autrichien considérait l'entrepreneur comme le moteur de la croissance économique, le seul capable de faire

\footnotetext{
18 A. Giuntini (dir.), Dati statistici, in Storia dell'Ansaldo. 2, cit., p. 238-241.

${ }^{19}$ E. Bagnasco, A. Rastelli, L'attività e la produzione cantieristica, cit. p. 90-107, Pour les données sur les tendances de l'emploi à Ansaldo, sur les investissements et désinvestissements dans les bâtiments et les machines, Relazione sull'andamento della Marina mercantile 1885-1887, cit., p. 244-247.

${ }^{20}$ Sur la crise bancaire et industrielle à Gênes et sur la position des industriels métallurgistes de la capitale de la Ligurie, G. Doria, Investimenti e sviluppo economico a Genova alla vigilia della prima guerra mondiale. 2, 1883-1914, Milan, Giuffrè, 1973, p. 94156, A. M. Falchero, Le strutture e l'evoluzione dell'Ansaldo, cit., p. 35.

${ }^{21}$ G. Giuntini, Dati statistici, cit., ad vocem.

22 Ici, nous renvoyons à la première analyse importante de l'économiste autrichien, SCHUMPETER J. A., Theorie der wirtschaftlichen Entwicklung, 1912; in inglese, The theory of economic development: an inquiry into profits, capital, credit interest and the business cycle, Oxford University Press, Oxford, 1934.
} 
des changements radicaux avec le renouvellement des produits ou des procédés, l'introduction de nouvelles matières premières, l'ouverture des marchés, les changements organisationnels et la structure du marché. L'entrepreneur de Schumpeter introduit de nouvelles combinaisons. C'est un homme d'action de l'économie, un leader actif qui recherche de nouvelles opportunités et de nouveaux marchés ${ }^{23}$.

Les frères Bombrini avaient de telles caractéristiques. Ces deux entrepreneurs ont cherché à assurer le succès de la Compagnie Ansaldo aux échelles nationale et internationale en augmentant sa surface. L'expansion internationale confirma le choix des frères Bombrini, entrepreneurs précurseurs de nouvelles stratégies de développement. La décision d'orienter l'entreprise vers les marchés étrangers était, à bien des égards également, une conséquence de l'efficacité technique acquise par les chantiers navals Ansaldo, grâce à laquelle l'entreprise était désormais en mesure de construire des croiseurs de la classe Garibaldi dans des délais rapides, «très différents des temps utilisés pour la Liguria et la Minerva, des croiseurs qui avaient également un tonnage beaucoup plus faible» ${ }^{24}$. Les croiseurs de la classe Garibaldi ont été construits en environ un an jusqu'au lancement, puis six mois pour les finitions. Le Liguria, conçu en 1889, ne fut livré que cinq ans plus tard. Le Minerva, malgré un tonnage modeste (843 tonnes de déplacement) nécessitait trois ans de travaux.

Les frères Bombrini avaient mis au point une organisation pour construire davantage d'unités navales du même type qui réduisait considérablement le temps. Cette structure de production offrait à Ansaldo la possibilité de concevoir et de mettre en œuvre avec succès des programmes transnationaux, une option que Giovanni Bombrini lui-même a défendu dans une lettre de mars 1898 à Ferdinando Maria Perrone, l'informant en bref de la décision de « nous mettre à la construction de nos propres navires de guerre ${ }^{25}$ :

Naturellement, nous devons tirer parti de ces éléments qui nous rendent supérieurs à tous nos concurrents, à savoir la qualité de la construction et de la conception, et surtout le manque de temps. Quant aux bâtiments, malheureusement les nôtres sont toujours trop beaux ; quant aux projets, nous sommes maintenant assez bien informés ; mais en ce qui concerne le peu de temps pour construire et équiper un navire, il faudrait recourir à des types déjà fabriqués. Certes, on peut finir un Garibaldi en quatorze mois, en quinze donner deux, en seize trois [...]. En réalisant notre rêve, nous arriverons à donner à la Compagnie Ansaldo une nouvelle position dans le monde et à défier tous les autres concurrents nationaux et étrangers, et nous aurions l'immense avantage de la liberté d'action ; et il n'y aurait plus de dépendance vis-à-vis d'un Brin ou à d'autres ingénieurs, qui essayeraient avec des moyens subtils de nous croiser sur notre chemin. De cette façon, nous nous imposerons également aux clients eux-mêmes pour notre façon de traiter les affaires et pour les résultats supérieurs de nos productions.

Les projets par Giovanni Bombrini, qui étaient partagés par son frère Carlo Marcello, d'investir le marché international montrent la confiance qu'il accordait à la réorganisation de la production de l'entreprise. Ils épousaient les tendances qui s'affirmaient dans l'industrie internationale de l'armement et paraissaient tout à fait réalisables. L'expansion à l'étranger a également été favorisée par la possibilité d'avoir un groupe d'agents et d'intermédiaires bien préparés d'un point de vue technique, relationnel et managérial. Parmi eux, Ferdinando Maria Perrone se distingue par son importance, probablement le plus grand architecte et le représentant le mieux accrédité à l'étranger de la politique transnationale d'Ansaldo, mais les frères Bombrini pouvaient également compter sur

\footnotetext{
${ }^{23}$ A. Salsano, Introduzione, in J. A. Schumpeter, L'imprenditore e la storia d'impresa. Scritti 1927-1949, Turin, Boringhieri, 1993, p. VIII.

24 A. M. Falchero, Le strutture e l'evoluzione dell'Ansaldo, cit., p. 37. Sur le processus évolutif de la chaîne de production de l'ingénierie navale de l'époque, G. Pedrocco, Alle origini della moderna navalmeccanica, in «Annali della Fondazione Giangiacomo Feltrinelli», XX, p. 951-972, (1979-1980).

25 Le document mentionné dans le texte se trouve dans les archives historiques de la Compagnie Ansaldo (à partir de maintenant ASA), dans l'Archive Perrone (à partir de maintenant AP), Divers, boîte 68, dossier 9.
} 
l'aide des autres collaborateurs comme Emanuele Giovanni Elia, Carmine Dellepiane, Nabor Soliani, Manuel Mario de Carvalho, qui ont contribué à accroître la présence de la société génoise sur le marché international des fournitures navales militaires.

\section{La conquête de l'Amérique du Sud et du Mexique : Ferdinando Maria Perrone, l'agent "stratégique " des frères Bombrini}

Ferdinando Maria Perrone (1847-1908) fut l'un des architectes du décollage international d'Ansaldo. En août 1895, il obtint des frères Bombrini la tâche de représenter la Compagnie Ansaldo en Amérique du Sud et au Mexique, avec un salaire fixe, le remboursement des dépenses et des pourcentages substantiels sur les ventes ${ }^{26}$. Sa mission reposait sur des bases solides. Il était un fin connaisseur de la réalité politique, économique et sociale de l'Amérique du Sud, en particulier de l'Argentine où il était arrivé avec sa famille en 1885. Il avait pu y nouer des relations importantes avec la bourgeoisie locale, avec la classe politique et avec le président Julio Alejo Roca, créant un important réseau clientéliste pour s'intégrer dans la structure d'un pays qui planifiait à l'époque un programme ambitieux de développement de l'industrie navale ${ }^{27}$. La position de Perrone au sein d'Ansaldo s'est encore renforcée quelques années plus tard. Avec l'accord signé le $1^{\text {er }}$ juillet 1901 et valable jusqu'au 27 juin 1904, la société de Gênes confie à Perrone « la gestion effective et exclusive des négociations et négociations concernant la vente des navires et produits de ses établissements à l'étranger », non seulement en Amérique du Sud mais aussi au Mexique. Perrone aurait reçu en 1901 une rémunération annuelle de 150000 francs or, ainsi qu'un pourcentage de 2 pour cent sur les bénéfices nets de chaque année de la société ${ }^{28}$.

Il utilisait le rôle de premier plan que lui avait confié sa collaboration avec les Bombrini pour développer une analyse de l'organisation globale d'Ansaldo à un stade critique de son existence et chercher des solutions pour accroitre la réussite de l'entreprise. À cet égard, il adopta la stratégie d'expansion des frères Bombrini vers les marchés étrangers, identifiant la clé du succès dans la figure de l'entrepreneur. Ces considérations ont été exprimées dans une lettre envoyée aux frères Bombrini, dans laquelle il montra une profonde adhésion au modèle d'entreprise qui inspirait la direction de l'entreprise ${ }^{29}$ :

Un établissement comme la Compagnie Ansaldo demande un travail acharné, un esprit éveillé, intuitif des choses et des hommes, une parfaite connaissance des événements qui se déroulent dans le monde, une attitude exceptionnelle face aux affaires, une vigilance sans pareil pour savoir les créer. À se tenir au courant de tout, grâce à une prodigieuse activité d'esprit et de personne et à un homme dont les pensées sont continuellement tournées vers les choses de la maison [Ansaldo]. Une usine comme Ansaldo, honneur et fierté de la nation, gloire et profit de la patrie pour le travail qu'elle représente, a besoin de se soutenir par une grande quantité de travail continu et hautement rémunérateur, afin de satisfaire tous les besoins de diverses natures (...). Il devrait embrasser le monde entier dans une concurrence impitoyable avec des semblables. Le combat est pour la vie. Il vaut mieux le chercher, le créer, le conquérir, le promouvoir et ne pas l'attendre. Vous et Carlotto êtes deux martyrs. Votre activité phénoménale ne suffit pas pour mener à bien la tâche de gestion. John doit même épuiser sa vie, emportant les plus belles heures de repos : les nocturnes. Je n'ai pas été compris.

Une telle intervention allait bien au-delà du partage de la stratégie d'entreprise des frères Bombrini. En fait, elle cachait la volonté de Ferdinando Maria Perrone de monter dans la hiérarchie

\footnotetext{
${ }^{26}$ P. Rugafiori, L'ascesa di Ferdinando Maria Perrone, in Storia dell'Ansaldo. 2. La costruzione, cit., p. 152.

${ }^{27}$ Ivi, p. 147-151.

28 ASA, AFB, b. 2, dossier 12, Contrat Bombrini-Perrone, mai 1901. Le contrat comprend un total de 10 articles.

29 ASA, AFB, b. 3, dossier 12, Perrone à Giovanni Bombrini ,30 avril 1901.
} 
de l'entreprise. Il aspirait à se hisser à la tête de l'entreprise, mais plus encore à la direction monolithique d'Ansaldo, objectif qui, d'ailleurs, s'est concrétisé à la suite de divers événements entre $1903-1904^{30}$.

Sa connaissance du continent latino-américain était appréciée par les Bombrini qui lui ont confié la mission de débloquer une impasse rencontrée au Brésil où depuis 1894 un entrepreneur local, Manuel Mario de Carvalho, était l'agent officiel d'Ansaldo. Malgré l'engagement abondant et le soutien, parfois clair, parfois dissimulé, de l'ancien président du Brésil Floriano Peixoto, Carvalho n'avait pu conclure aucun contrat de vente d'unités navales ${ }^{31}$. Au cours de sa présidence, avant la prise de fonctions de Carvalho, la Compagnie Ansaldo avait réussi à construire une " usine », une sorte de structure potentielle, destinée à abriter la base des opérations de l'Ansaldo.

En acceptant le tâche, Perrone n'eut aucune difficulté à confirmer les prévisions pessimistes de Carvalho aux frères Bombrini : le Brésil était un marché difficile en raison de la grave crise financière et économique. Malgré cela, Perrone avait tout de même accepté l'invitation et avait décidé de rester quelques jours au Brésil ${ }^{32}$. Selon toute vraisemblance, sa décision dépendait du fait qu'il savait que le gouvernement brésilien s'apprêtait à moderniser la flotte dans un proche avenir, à la suite du message d'ouverture du Congrès du Président Campo Salles. On ne savait pas encore comment le gouvernement brésilien allait financer le projet, mais Perrone jugeait indispensable de se rendre à Rio de Janeiro le plus tôt possible pour se faire une idée générale de la situation ${ }^{33}$.

Il ne semble pas que pendant le period de Perrone (1901-1904), la Compagnie Ansaldo ait pu approvisionner la flotte de guerre brésilienne. Il est très probable qu'il ait rencontré les mêmes difficultés qui ont été à l'origine de l'échec de Carvalho. En particulier, la forte concurrence des compagnies maritimes françaises, anglaises, écossaises et allemandes, ainsi que les relations politiques beaucoup plus efficaces et prometteuses de ces dernières avec le gouvernement brésilien, semblent avoir joué un rôle déterminant ${ }^{34}$.

Contrairement au Brésil, Perrone avait obtenu de trés bons résultats en Argentine :

En juillet 1895 [un mois avant que Perrone ne soit nommé représentant officiel de la Compagnie Ansaldo pour l'Amérique du Sud], le gouvernement argentin achète le croiseur blindé Garibaldi à Ansaldo grâce à la médiation de Perrone. Une déclaration d'une importance absolue puisqu'elle a été obtenue sur un marché jusque-là inconnu et en concurrence directe avec de grandes entreprises étrangères, fort d'un soutien économique et politique bien supérieur à celui fourni par le fragile État italien de Crispina. Une série de circonstances favorables sont intervenues en faveur de la société génoise, de la détérioration progressive des relations entre l'Argentine et le Chili, aux délais de livraison rapides du navire en construction pour le gouvernement italien prêt à y renoncer. Mais ces facteurs n'auraient guère conduit au succès de l'accord sans l'intervention décisive par Perrone $e^{35}$.

De plus, l'idée de vendre le Garibaldi à l'Argentine, avec les autres facteurs déjà mis en évidence, a convaincu les frères Bombrini d'accélérer la nomination de Perrone en tant que représentant officiel en Amérique du Sud. Dans les années 1896-1901, ce dernier enregistra un autre succès important : la Compagnie Ansaldo a été chargée par le gouvernement argentin de fournir trois croiseurs, un autre croiseur de la classe Garibaldi, avait été commandé par le gouvernement espagnol. Après sa nomination en juillet 1901 comme directeur et représentant général de la société

30 M. Doria, I Perrone e l'Ansaldo nella storia d'Italia in Fondazione Ansaldo, Archivio Perrone 1871-1945. Guida all'inventario, Gênes, Fondation Ansaldo, 2011, p. 18-19.

31 ASA, AFB, b. 5, dossier 6, Carvalho aux frères Bombrini, 22 dicembre 1894.

32 Ivi, b. 2, dossier 12, Perrone aux frères Bombrini, 30 avril 1901.

33 Ivi, b. 3, dossier 12, Perrone aux frères Bombrini, 14 mai 1901.

${ }^{34}$ Ivi, b. 5, dossier 6, Carvalho aux frères Bombrini, 10, 24 avril et 9 mai 1895.

${ }^{35}$ P. Rugafiori, L'ascesa di Ferdinando Maria Perrone, cit., p. 152. 
à l'étranger, son rôle a été important dans la politique d'expansion de la Compagnie Ansaldo, d'autant plus qu'il participa aux approvisionnements les plus importants de navires militaires à l'étranger, comme par exemple, le contrat pour la fourniture au Japon en 1902 des croiseurs blindés de type Kasuga et Nisshin et pour la fourniture à la Turquie du navire cuirassé Messudieb et de trois corvettes blindées ${ }^{36}$.

\section{A la conquête de l'Angleterre, des Pays-Bas et de la Belgique : Emanuele Giovanni Elia (1866-1935), l'agent « inventeur » des frères Bombrini}

Dans les projets internationaux des frères Bombrini, Emanuele Giovanni Elia peut être considéré comme le facteur gagnant pour la Compagnie Ansaldo qui plaçait beaucoup de confiance et d'espoir dans ses compétences techniques et professionnelles incontestables. Excellent ingénieur d'armes sous-marines, il a inventé la torpille bloc qui porte son nom (ainsi que d'autres engins), utilisée par les Alliés pendant la Première Guerre mondiale. Avant de l'invention par d'Elia, la mise en œuvre de la torpille nécessitait des opérations longues, laborieuses et coûteuses. Au contraire, avec le modèle qu'il a conçu et testé en 1890, la mise en œuvre devenait une opération facile, même en haute mer ; de sorte qu'il était possible d'utiliser la torpille d'une manière « offensive », c'est-à-dire capable de viser les mouvements de l'ennemi et non plus seulement de défendre les côtes ou les forteresses ${ }^{37}$. L'innovation fondamentale consistait dans les mécanismes de positionnement automatique de la torpille à la profondeur désirée. Grâce à ces automatismes, la vitesse d'installation augmenta considérablement : en 1895 un navire porte-mine pouvait placer 200 torpilles Elia en deux heures et avec 100 matelots, alors qu'auparavant la pose de la même quantité nécessitait deux mois et un millier de matelots. La torpille était équipée d'un dispositif de sécurité (une plaque hydrostatique), qui empêchait l'allumage de la charge explosive en cas de rupture du flacon d'électrolyte suite à un impact accidentel sur le pont du navire porte-mine. La torpille fuit officiellement adoptée par la marine italienne entre 1893 et 1899.

Elia fut envoyé par les Bombrini en mission en Angleterre avec de sérieux espoirs de succès car l'agent de la Compagnie Ansaldo aurait proposé à la marine anglaise, alors la plus puissante du monde, une importante unité navale de combat qu'elle ne possédait pas. Le contrat exclusif évitait la concurrence avec les puissantes compagnies maritimes britanniques et américaines. Il prévoyait 10 torpilleurs en six semaines, 500 en 55 mois, pour les torpilleurs restants selon une subdivision de 75 unités par mois. Le paiement aurait été effectué avec 25000 livres à la signature du contrat puis au taux de 1500 livres pour chaque lot de 25 torpilleurs. Elia obtint un succès encore plus grand en fournissant à la flotte navale anglaise une nouvelle arme de sa propre invention, une mine sousmarine à installer sur les cuirassés, ainsi que les torpilles bloc, nécessaires au montage de la mine ${ }^{38}$. Une première fourniture de dix mines à tester sans charge explosive, pour un coût de 8000 livres, a été suivie d'une seconde fourniture de 500 mines dans l'année suivant la signature du contrat, au prix de 55000 livres sterling ${ }^{39}$.

Parallèlement, Elia édifia un réseau de sociétés auxiliaires autour de Torpedini Management d'Ansaldo, dans le but d'améliorer l'offre de l'entreprise à l'étranger, ainsi que d'augmenter le potentiel des entreprises du groupe. Dans ce contexte, un accord a été signé avec la Compagnie Glisenti de Brescia qui a construit des fusils semi-automatiques à tir sélectif qui avaient été créés par Amerigo Cei-Rigotti en 1890 et dans la version finale en 1900. Un contrat substantiel fut signé avec le gouvernement anglais pour ces fusils. Cette opération a été facilitée par l'intérêt de Glisenti pour

\footnotetext{
${ }^{36}$ Ivi, p. 154-158 et A. Giuntini, Dati statistici, ivi, p. 240.

${ }^{37}$ Pour une biographie exhaustive concernant Elia, W. Polastro, Elia, Giovanni Emanuele in Dizionario Biografico degli Italiani, Rome, Institut de l'Encyclopedie Italienne, 1993, vol. 42, ad vocem.

${ }^{38}$ ASA, AFB, b. 7, dossier 7, Elia aux frères Bombrini, Londres 3 septembre 1900.

${ }^{39}$ Ivi, Compagnie Ansaldo au Secrétariat de l'Amirauté britannique, 3 septembre 1900, Elia aux frères Bombrini, 12septembre 1900.
} 
le dispositif de déclenchement des torpilles Elia, réalisant ainsi la fusion de deux inventions ingénieuses en une encore plus meurtrière.

L'activité commerciale d'Elia aux Pays-Bas fut tout aussi intense. Les frères Bombrini avaient conseillé à l'ingénieur de s'installer à La Haye pour conduire les négociations. La marine néerlandaise avait besoin de se moderniser considérablement ${ }^{40}$. Elle prévoyait une dépense de 4200 000 florins répartis en dix ans et Ansaldo devait fournir 35 torpilleurs blocs pour un coût total de plus de 122000 florins $^{41}$. Le renforcement de la flotte navale pour défendre l'immense empire colonial néerlandais était aussi envisagé. L'immensité des espaces à couvrir appelait l'utilisation de navires propulsés au pétrole et non au charbon, ce qui n'était possible qu'en achetant le brevet par Vittorio Cuniberti ${ }^{42}$. Grâce à Elia, Ansaldo réussit à transformer un navire au charbon en un navire au mazout, de sorte qu'elle a pu commencer à fournir la flotte néerlandaise. Dans le même temps, il avait empêché la société concurrente des frères Orlando de construire la nouvelle unité navale sous un régime de monopole en acquérant le brevet par Cuniberti ${ }^{43}$.

Elia avait aussi réussi à inclure la Compagnie Ansaldo dans le projet de modernisation du port de Rotterdam. Van Gelder, l'un des ingénieurs navals les plus renommés de la marine néerlandaise, avait déclaré à plusieurs reprises que l'investissement de capitaux étrangers dans l'industrie navale des Pays-Bas était non seulement nécessaire, mais souhaitable pour une série de raisons techniques et commerciales ; raisons que la Compagnie Ansaldo était alors en mesure de satisfaire ${ }^{44}$. Les négociations pour la fourniture de torpilles bloc conçues par Elia avaient été engagées en mai 1900 à la suite du rapport favorable de la Commission de la marine néerlandaise sur les essais effectués à Gênes et à Messine. Les Pays-Bas ont ainsi renforcé leur flotte de guerre en acquérant six torpilleurs et des torpilles de la Compagnie Ansaldo ${ }^{45}$. Elia a su comprendre et s'approprier les ressorts de la politique économique et commerciale des Pays-Bas et s'y adapter.

Ce succès poussa les frères Bombrini à lui confier également le marché belge. Un premier accord avec la Belgique remonte à septembre 1901 pour la fourniture de dix torpilles pour un coût total de 200000 francs, à destination de la colonie du Congo. Les négociations entamées dans la première partie de l'année se sont terminées de manière inattendue à la fin de l'année, à tel point qu'Elia a été contraint de rentrer à Bruxelles depuis les États-Unis où il était parti pour conclure d'autres affaires. Un autre accord fut conclu en août 1902 pour la fourniture de dômes blindés et d'armes pour la défense terrestre et maritime au gouvernement belge après des négociations entamées en juillet avec le général Docteur ${ }^{46}$. La commande portait sur environ 205 dômes pour un coût total d'environ de150000 000 francs.

L'autre projet commandé à Elia par le gouvernement belge pour augmenter la capacité de défense du port d'Anvers concernait la construction d'un «barrage à marée basse, moyenne et haute ${ }^{47}$. La largeur totale du barrage, de 1190 mètres, était «bien défendable de la batterie et dans des conditions optimales [par rapport à] Cherbourg et Portsmouth ainsi qu'à New York [où il y avait]

\footnotetext{
${ }^{40}$ Rapport provisoire de la Commission «sur le projet de loi formant le VI Chapitre du budget de l'Etat pour l'année 1900 »

${ }^{41}$ Ivi, dossier 5, Première Chambre des États généraux de la Compagnie Ansaldo, 30 janvier 1900, b. 7, dossier 8, Ministre de la Marine Néerlandaise aux frères Bombrini, 26 octobre 1900.

42 Vittorio Cuniberti (1854-1913), colonel de la Marine, il était un brillant concepteur de cuirassés, dont le Dante Alighieri, le premier cuirassé mono-calibre de la marine de guerre italienne.

${ }^{43}$ Ibidem. Concernant Cuniberti, Dizionario biografico degli italiani, Rome, Institut de l'Encyclopedie Italienne, 1985, vol. 31, ad vocem

${ }^{44}$ Ivi, dossier 4, Mémorandum relatif à la construction d'un nouveau chantier naval à Rotterdam, rédigé par Gelder et envoyé par Elia aux frères Bombrini le 20 janvier 1900.

${ }^{45}$ Ivi, b. 7, dossier 8, Elia al ministro delle Colonie e della Marina Olandese, 21 dicembre 1900, dossier 9, Elia aux frères Bombrini, 2 dicembre 1900.

${ }^{46}$ Ivi, dossier 7, Elia aux frères Bombrini, 17 agosto 1902.

47 Ivi, Elia à Briffaux, 24 mars 1903.
} 
des barrages à 6000 mètres des canons de protection ». Dans le cas particulier du port d'Anvers « pas un seul navire ne pouvait franchir un système à triple barrière comme celui en question ${ }^{48}$. Elia ajoutait que : « car les navires de guerre plus petits qui n'ont pas de blindage comme les Pays-Bas ont déjà un tirant d'eau de 6 mètres, alors qu'en cas de marée haute, le barrage le plus bas, qui est à 4,50 mètres, entrerait déjà en action. Après tout (poursuit Elia), il n'y a pas de vaisseau blindé qui puisse défiler devant un fort sans risque de retournement de moins de 5 mètres. Si au lieu de mettre les torpilles à 70 mètres, on adopte la distance de 50 mètres, on aurait un barrage de 50 x $3=150 \mathrm{~m}$. x $3=450$ mètres et $450+50 \times 2=550$ mètres pour toute la défense. En supposant que la marée soit à sa hauteur maximale, nous aurions le barrage de mer le plus proche possible à 4,50 mètres d'immersion, le deuxième à 3,50 mètres, le troisième à 2,50 mètres. À marée basse, la première barrière se situerait à 0,50 mètre sous l'eau et les deux autres à la surface ${ }^{49}$. En fin de compte, Elia avait proposé de ne pas modifier la torpille de son invention et d'exploiter l'avantage qu'Ansaldo avait déjà acquis, en évitant d'engager des dépenses pour les tests et les essais auxquels les autres nations devaient nécessairement faire face.

\section{Quelques remarques finales}

Les frères Bombrini étaient des entrepreneurs dynamiques et ouverts d'esprit. Ils ont tenté de trouver des solutions avantageuses pour l'entreprise Ansaldo alors que la situation économique et financière de la fin du XIXe siècle en Italie avait plongé le secteur de l'ingénierie navale dans la crise. Ils ont été les promoteurs de l'expansion et de la recherche de nouveaux marchés dans lesquels ils ont pénétré malgré les difficultés en offrant des produits de nouvelle génération issus de l'industrie militaire et navale. Les frères Bombrini ont fait appel à un réseau de collaborateurs experts et compétents, qui étaient de fins connaisseurs des marchés et des dynamiques qui y étaient liés. Ils ont incarné le modèle schumpétérien de l'entrepreneur : ils ont su exploiter les inventions et les transformer en innovations, sentant les possibilités de profit, « ... l'innovation technique, calculée dans les moindres détails, lui est imposée par ses ingénieurs. Ce regard, ce don de divination, a été remplacé par les calculs du spécialiste », mais surtout, ils étaient des explorateurs audacieux de nouveaux marchés à la recherche de débouchés commerciaux sans limites géographiques, culturelles et sociales ${ }^{50}$.

La tentative des frères Bombrini de se développer sur les marchés étrangers constitua un projet ambitieux pour l'entreprise qui se trouvait confrontée à de grandes sociétés internationales proposant des produits à des prix bas et compétitifs. Ils ont persévéré dans la recherche de nouvelles opportunités et de nouvelles connexions, tissant des relations diplomatiques et de clientèle avec les environnements économico-politiques des pays étrangers. Les résultats bien que positifs n'ont pas été suffisants pour imposer Ansaldo sur la scène internationale. Toutefois, la politique d'expansion des frères Bombrini a représenté un exemple valable pour les directeurs ultérieurs de l'entreprise, qui ont continué à planifier la stratégie d'entreprise vers le développement à l'étranger.

\section{Sources d'archives}

Archivio Storico Ansaldo (à partir de maintenant ASA), Archivio Perrone (à partir de maintenant AP), Divers, boîte 68, dossier 9.

ASA, AFB, b. 2, dossier 12, Contrat Bombrini-Perrone, mai 1901.

\footnotetext{
${ }^{48}$ Ivi, Rapport par Elia à la Commission royale, ler août 1903.

${ }^{49}$ Ibidem.

50 J. A. Schumpeter, Le classi sociali in ambiente tecnicamente omogeneo in Id., «Sociologia dell'imperialismo». Laterza, Bari, 1974 , p. 65.
} 
ASA, AP, b. 3, dossier 12, Perrone à Giovanni Bombrini, 30 avril 1901.

ASA, AFB, b. 5, dossier 6, Carvalho aux frères Bombrini, 22 décembre 1894.

ASA, AFB, b. 2, dossier 12, Perrone aux frères Bombrini, 30 avril 1901.

ASA, AP, b. 3, dossier 12, Perrone aux frères Bombrini, 14 mai 1901.

ASA, AFB, b. 5, dossier 6, Carvalho aux frères Bombrini, 10, 24 avril et 9 mai 1895.

ASA, AFB, b. 7, dossier 7, Elia aux frères Bombrini, Londres 3 septembre 1900, Compagnie Ansaldo au Secrétariat de l'Amirauté britannique, 3 septembre 1900, Elia aux frères Bombrini, 12 septembre 1900.

ASA, AFB, b. 7, dossier 7, Elia aux frères Bombrini, Londres 3 septembre 1900.

ASA, AFB, Compagnie Ansaldo au Secrétariat de l'Amirauté britannique, 3 septembre 1900, Elia aux frères Bombrini, 12 septembre 1900.

ASA, AFB, dossier 5, Première Chambre des États généraux de la Compagnie Ansaldo, 30 janvier 1900,

ASA, AFB, b. 7, dossier 8, Ministre de la Marine Néerlandaise aux frères Bombrini, 26 octobre 1900.

ASA, AFB, dossier 4, Mémorandum relatif à la construction d'un nouveau chantier à Rotterdam, compilé par Gelder et envoyé par Elia aux frères Bombrini le 20 janvier 1900.

ASA, AFB, b. 7, dossier 8, Elia au ministre des Colonies et de la Marine Néerlandaise, 21 décembre 1900, dossier 9, Elia aux frères Bombrini, 2 décembre 1900.

ASA, AFB, b. 8, fas. 3, Elia aux frères Bombrini, Bruxelles, 18 septembre 1901, Washington 17 octobre, 1901 et New York, 2 novembre 1901.

ASA, AFB dossier 7, Elia aux frères Bombrini, 17 août 1902.

ASA, AFB, Elia à Briffaux, 24 mars 1903.

ASA, AFB, Rapport par Elia à la Commission royale, 1er août 1903.

\section{Bibliographie}

AA. VV., La Breda. Dalla Società Italiana Ernesto Breda alla Finanziaria Ernesto Breda 1886-1986, Amilcare Pizzi Editore, Milan, 1986.

AMATORI F., COLLI A., Impresa e industria in Italia dall 'Unità a oggi, Marsilio, Venise, 1999.

BAGNASCO E., RASTELLI A., L'attività e la produzione cantieristica, in MORI G. (dir.), Storia dell 'Ansaldo. 2. La costruzione di una grande impresa 1883-1902, Laterza, Bari, 1995.

BARONE G., «Lo Stato e la Marina Mercantile in Italia (1881-1882)» in Studi Storici, n. 3, p. 624-659, 1974.

BONELLI F., Lo sviluppo di una grande impresa in Italia. La Terni dal 1884 al 1962, Einaudi, Turin, 1975.

BOZZONI A., Relazione della Commissione per le industrie meccaniche e navali, Tipografia Fratelli Bencini, Rome, 1885.

BOZZONI A., Relazione a Sua Eccellenza e il mare. Costruzioni navali, trasporti e commercio tra XV e XX secolo,

Tipografia Fratelli Bencini, Rome, 1889.

BOZZONI G., Marina militare e costruzioni navali, in Cinquant'anni di storia italiana, Hoepli, Milan, 1911. 
CIOCCA P., TONIOLO G., Storia economica d 'Italia, 2, Annali, Laterza, Bari, 1999.

COMPAGNIE ANSALDO, Notizie sullo stabilimento meccanico e di costruzioni navali Gio Ansaldo e C. di Sampierdarena con breve cenno sugli oggetti da esso presentati alla Esposizione Generale di Torino, Turin, 1884.

D'AQUINO U., «Cuniberti Vittorio Emilio», in Dizionario biografico degli italiani, Institut de l'Encyclopedie Italienne, Rome, 1985, vol. 31, ad vocem.

DE COURTEN L., «L 'Ansaldo e politica navale», in Storia dell 'Ansaldo. Dal crollo alla ricostruzione 1919-1929, G. DE ROSA (dir.), 5 vol., Laterza, Bari, 1998.

DE ROSA L., Difesa militare e sviluppo economico in Italia (1861-1914), in L. DE ROSA, La rivoluzione industriale in Italia e il Mezzogiorno, Laterza, Rome-Bari, 1973.

DORIA G., Investimenti e sviluppo economico a Genova alla vigilia della prima guerra mondiale. 2, 1883-1914, Giuffrè, Milan, 1973.

DORIA M., «I Perrone e l'Ansaldo nella storia d'Italia» in Fondation Ansaldo, Archivio Perrone 1871-1945. Guida all'inventario, Fondation Ansaldo, Gênes, 2011.

DORIA M., L’Ansaldo. L'impresa e lo Stato, Franco Angeli, Milan, 1989.

FALCHERO A. M., Le strutture e l'evoluzione dell 'Ansaldo, in MORI G. (dir.), Storia dell 'Ansaldo. 2. La costruzione di una grande impresa 1883-1902, Laterza, Bari, 1995.

GIUNTINI A., Dati statistici, in MORI G., (dir.), Storia dell 'Ansaldo. 2. La costruzione di una grande impresa 18831902, Laterza, Bari, 1995.

HERTNER P., «Capitale tedesco e industria meccanica in Italia: la Esslingen a Saronno», in Società e Storia, n. 17, p. 583-621, 1982.

LICINI S., «Dall Elvetica alla Breda. Alle origini di una grande impresa milanese (1846-1918)», in Società e Storia, $\mathrm{n}$. 63, p. 79-123, 1994.

LUNGONELLI M., «L 'Ansaldo e 1 'industria metalmeccanica italiana» in MORI G., (dir.), Storia dell 'Ansaldo. 2. La costruzione di una grande impresa 1883-1902, Laterza, Bari, 1995.

MERGER M., «L'industrie italienne de locomotives, reflet d'une industrialisation tardive et difficile (1850-1914)», in Histoire Economie et Societè, n. 3, p. 335-370, 1989.

MERGER M., «Un modello di sostituzione: la locomotiva italiana dal 1850 al 1914», in Rivista di Storia Economica, (III), n. 1, pp. 66-105, 1986.

MORI G., (dir.), Storia dell 'Ansaldo. 1. Le origini 1853-1882, Laterza, Bari, 1994.

PEDROCCO G., «Alle origini della moderna navalmeccanica» in Annali della Fondazione Giangiacomo Feltrinelli, XX, p. 951-972, 1979-1980.

POLASTRO W., Elia Giovanni Emanuele in Dizionario Biografico degli Italiani, Roma, Institut de l'Encyclopedie Italienne, vol. 42, 1993, ad vocem.

RUGAFIORI P., Ferdinando Maria Perrone: da Casa Savoia all ’Ansaldo, Utet, Turin, 1992.

RUGAFIORI P., «L 'ascesa di Ferdinando Maria Perrone», in MORI G. (dir.), Storia dell 'Ansaldo. 2. La costruzione di una grande impresa 1883-1902, Bari, Laterza, 1995.

SALSANO A., Introduzione, in J. A. Schumpeter, L’imprenditore e la storia d'impresa. Scritti 1927-1949, Boringhieri, Turin, 1993.

SCHUMPETER J. A., «Le classi sociali in ambiente tecnicamente omogeneo» in Id., Sociologia de l'imperialismo, Laterza, Bari, 1974.

SCHUMPETER J. A., Theorie der wirtschaftlichen Entwicklung, 1912; in inglese, The theory of economic development: an inquiry into profits, capital, credit interest and the business cycle, Oxford University Press , Oxford, 1934. 